\title{
Double exchange bias in ferrimagnetic heterostructures
}

\author{
B. Hebler, ${ }^{1}$ P. Reinhardt, ${ }^{2}$ G. L. Katona,${ }^{3}$ O. Hellwig, ${ }^{4,}{ }^{*}$ and M. Albrecht ${ }^{1}$ \\ ${ }^{1}$ Institute of Physics, University of Augsburg, Universitätsstraße 1, D-86159 Augsburg, Germany \\ ${ }^{2}$ Institute of Physics, Technische Universität Chemnitz, Reichenhainer Straße 70, D-09107 Chemnitz, Germany \\ ${ }^{3}$ Department of Solid State Physics, University of Debrecen, H-4010 Debrecen, P.O. Box 2, Hungary \\ ${ }^{4}$ San Jose Research Center, HGST a Western Digital Company, 3403 Yerba Buena Road, San Jose, California 95135, USA
}

(Received 7 November 2016; published 10 March 2017)

\begin{abstract}
We report on the magnetic reversal characteristics of exchange coupled ferrimagnetic (FI) $\mathrm{Tb}_{19} \mathrm{Fe}_{81} / \mathrm{Tb}_{36} \mathrm{Fe}_{64}$ heterostructures. Both layers are amorphous and exhibit strong perpendicular magnetic anisotropy. The investigated heterostructures consist of a Tb-dominated and a Fe-dominated FI layer. Thus, in the magnetic ground state the net moments of the individual layers are oppositely aligned due to antiferromagnetic coupling of $\mathrm{Fe}$ and $\mathrm{Tb}$ moments. By cooling the system below $160 \mathrm{~K}$, a large positive and negative exchange bias (EB) effect appears for the Tb- and Fe-dominated layers, respectively. The biasing depends only on the initial magnetization state and is neither affected by a cooling field nor by loop cycling. The phenomenon can be explained by the presence of a hard magnetic Fe-dominated interfacial layer, which forms during the sputter deposition process due to interface mixing and resputtering effects. This interfacial layer acts as a pinning layer below a certain temperature, where its coercivity increases to values larger than the accessible magnetic field range. This assumption is further supported by introducing a 0.9-nm-thick Ru spacer layer, which causes the EB effect to vanish. The EB effect was further investigated for a sample series, where the thickness ratio of the two Tb-Fe layers was varied, while keeping the total thickness of the bilayers constant. Only samples where the individual layers are sufficiently thick reveal double shifted loops, indicating the high sensitivity of the observed bias effect with respect to the magnetic properties of the individual layers and their interfacial area.
\end{abstract}

DOI: 10.1103/PhysRevB.95.104410

\section{INTRODUCTION}

The exchange bias (EB) effect, which refers to the shift of the hysteresis loop of a ferromagnet (FM) in direct contact to an antiferromagnet (AFM), has been investigated intensively over a period of 60 years [1-9] after its discovery by Meiklejohn and Bean [1]. In addition to AFM/FM interfaces, EB has also been reported in systems with ferrimagnetic (FI)/FM layers [10-23], resulting in giant $\mathrm{EB}$ fields in the order of several tens of kilo-Oersteds [13,14,24-26]. In particular, FI layers consisting of amorphous heavy rare earth (RE)-3d transition metal (TM) alloys provide further benefits as a pinning layer since these alloys can exhibit large interfacial exchange interaction and zero moment at the compensation temperature $T_{\text {comp }}$. Below this temperature, the heavy RE magnetization dominates, while above $T_{\text {comp }}$, the TM magnetization is the leading one. Thus, these FI alloys offer the possibility to change the magnetic properties by composition and temperature.

The EB effect of coupled hard and soft magnetic bilayers, which is based on an exchange spring behavior [27], can only be observed if the full hysteresis loop shows the reversal of the individual layers at different fields. Depending on the magnetic properties of both layers, the shift of the hysteresis can be in positive or negative field direction under minor loop conditions. Considering a heterostructure, consisting of two FI layers, where one layer is RE dominated and the other is TM dominated, in the ground state, the net moments of each

\footnotetext{
*Present address: Institute of Ion-Beam Physics and Materials Research, Helmholtz-Zentrum Dresden-Rossendorf, D-01314 Dresden, Germany; Institute of Physics, Technische Universität Chemnitz, Reichenhainer Straße 70, D-09107 Chemnitz, Germany.
}

layer are antiparallel aligned. However, by reversing the softer layer against the hard layer, an interfacial domain wall (IDW) at the transition region between the two layers will be created $[11,14]$. Reducing the external field leads to annihilation of the IDW, forcing the softer layer to rotate back which results in a positive loop shift under this minor loop condition. If the two coupled layers exhibit the same dominating elements (RE/RE or TM/TM), a negative EB occurs. These two cases have been recently reported for $\mathrm{DyCo}_{5} / \mathrm{Fe}_{76} \mathrm{Gd}_{24}$ heterostructures [19]. In other recent papers, large EB has been observed in FI $\mathrm{Ru}_{0.25} \mathrm{Cr}_{0.75} \mathrm{O}_{2}$ thin films [28] and FI Heusler alloys [29]. In all these cases, the EB effect is only observed for minor loop reversal, meaning that the pinning layer will not be reversed in the applied field range.

In this paper, the reversal characteristics of exchange coupled FI $\mathrm{Tb}_{19} \mathrm{Fe}_{81} / \mathrm{Tb}_{36} \mathrm{Fe}_{64}$ heterostructures, consisting of a Tb-dominated and a Fe-dominated FI layer with perpendicular magnetic anisotropy, are investigated. Most striking, below $160 \mathrm{~K}$, two simultaneously shifted hysteresis loops of different shape and magnitude occur in positive and negative field directions. We show that the observed effect requires a certain thickness ratio of the individual FI layers. Furthermore, its dependence on temperature, magnetic domain configuration, and cooling field is presented.

\section{EXPERIMENTAL DETAILS}

Ferrimagnetic $\quad \mathrm{Tb}_{19} \mathrm{Fe}_{81}(20 \mathrm{~nm}-x) / \mathrm{Tb}_{36} \mathrm{Fe}_{64}(x)$ thin films with $x=5,7.5,10,12.5$, and $15 \mathrm{~nm}$, exhibiting strong perpendicular magnetic anisotropy, were prepared by dc magnetron cosputtering at room temperature on $\mathrm{SiO}_{2}(100 \mathrm{~nm}) /$ $\mathrm{Si}(001)$ substrates. The base pressure of the deposition chamber was below $8 \times 10^{-8}$ mbar, and during the deposition, 
an Ar pressure of $1.5 \times 10^{-3}$ mbar (up to $5.0 \times 10^{-3} \mathrm{mbar}$ ) was used. The sample holder rotates during the deposition to get a uniform film composition. To improve film growth, a $\operatorname{Pt}(5 \mathrm{~nm}) / \mathrm{Tb}(2 \mathrm{~nm})$ seed layer system was used. In addition, all samples were covered with a 5-nm-thick Pt cap layer to prevent oxidation of the $\mathrm{Tb}$-Fe layers.

The composition of the alloys and the thickness of each layer in the film stack were determined by Rutherford backscattering spectrometry (RBS) using $1.7 \mathrm{Me} \mathrm{He}^{+}$ions at a scatter angle of $170^{\circ}$. Furthermore, composition-depth profiles of selected samples were extracted by secondary neutral mass spectrometry (SNMS) with a depth resolution of $<2 \mathrm{~nm}$. We used the direct bombardment mode with inductively coupled low-pressure radiofrequency Ar plasma, serving as a source of bombarding ions and also as a postionization medium [30]. The sputtering area was $3 \mathrm{~mm}$ in diameter. The structural properties were analyzed by $\mathrm{x}$-ray diffraction (XRD), confirming that all prepared alloys are amorphous (not shown).

The integral magnetic properties of the samples were determined using superconducting quantum interference devicevibrating sample magnetometry (SQUID-VSM). In addition, the magnetic properties of the individual FI layers of a $\mathrm{Tb}_{19} \mathrm{Fe}_{81}(10 \mathrm{~nm}) / \mathrm{Tb}_{36} \mathrm{Fe}_{64}(10 \mathrm{~nm})$ film sample were studied by soft $\mathrm{x}$-ray magnetic circular dichroism (XMCD) [31]. To allow for sufficient transmission of soft $\mathrm{x}$-rays, this film sample was prepared on a 200 -nm-thick $\mathrm{Si}_{3} \mathrm{~N}_{4}$ membrane. In transmission geometry, the signals are proportional to the projection of the magnetization onto the photon propagation direction, therefore providing maximum sensitivity to perpendicular magnetized films. Element-specific hysteresis loops were measured at the $\mathrm{Fe} L_{3}$ edge $(708 \mathrm{eV})$ and $\mathrm{Tb} M_{5}$ edge $(1241 \mathrm{eV})$ up to a maximum external field of $\pm 80 \mathrm{kOe}$ in the temperature range from room temperature down to $40 \mathrm{~K}$. The XMCD absorption experiments were performed at the high-field end station VEKMAG installed at the PM2 beamline of the Helmholtz-Zentrum Berlin (HZB).

\section{RESULTS AND DISCUSSION}

Remanent magnetization $M_{\mathrm{R}}$ vs temperature $\left(M_{\mathrm{R}}-T\right)$ measurements were carried out for the two 10-nm-thick Tb-Fe single layers employed in this paper. The following procedure was applied: First, the sample was saturated at room temperature in a perpendicular magnetic field of $+70 \mathrm{kOe}$. Then the sample was cooled down to $4 \mathrm{~K}$ in zero field (ZFC). Thereafter, the temperature was again ramped from 4 to $350 \mathrm{~K}$, and the remanent magnetization perpendicular to the sample plane was recorded. The $M_{\mathrm{R}}-T$ curves are shown in Fig. 1(a). As expected, both layers do not show a compensation temperature for these compositions and stay either $\mathrm{Tb}$ or $\mathrm{Fe}$ dominant $[32,33]$. We also determined the uniaxial magnetic anisotropy constant $K_{\mathrm{U}}$ of both layers as a function of temperature using the expression $K_{\mathrm{U}}=\frac{M_{\mathrm{S}} \cdot H_{\mathrm{A}}}{2}+2 \cdot \pi \cdot M_{\mathrm{S}}^{2}$, which takes the shape anisotropy contribution into account. While for the Fe-dominated sample, a more or less constant anisotropy value is obtained, the Tb-dominated sample shows smaller values, which increase strongly toward lower temperatures [Fig. 1(b)]. This behavior is basically given by the temperature dependence of $M_{\mathrm{R}}$. The temperature dependence of the coercivity $H_{\mathrm{C}}$ is shown in Fig. 1(c), revealing a similar behavior for
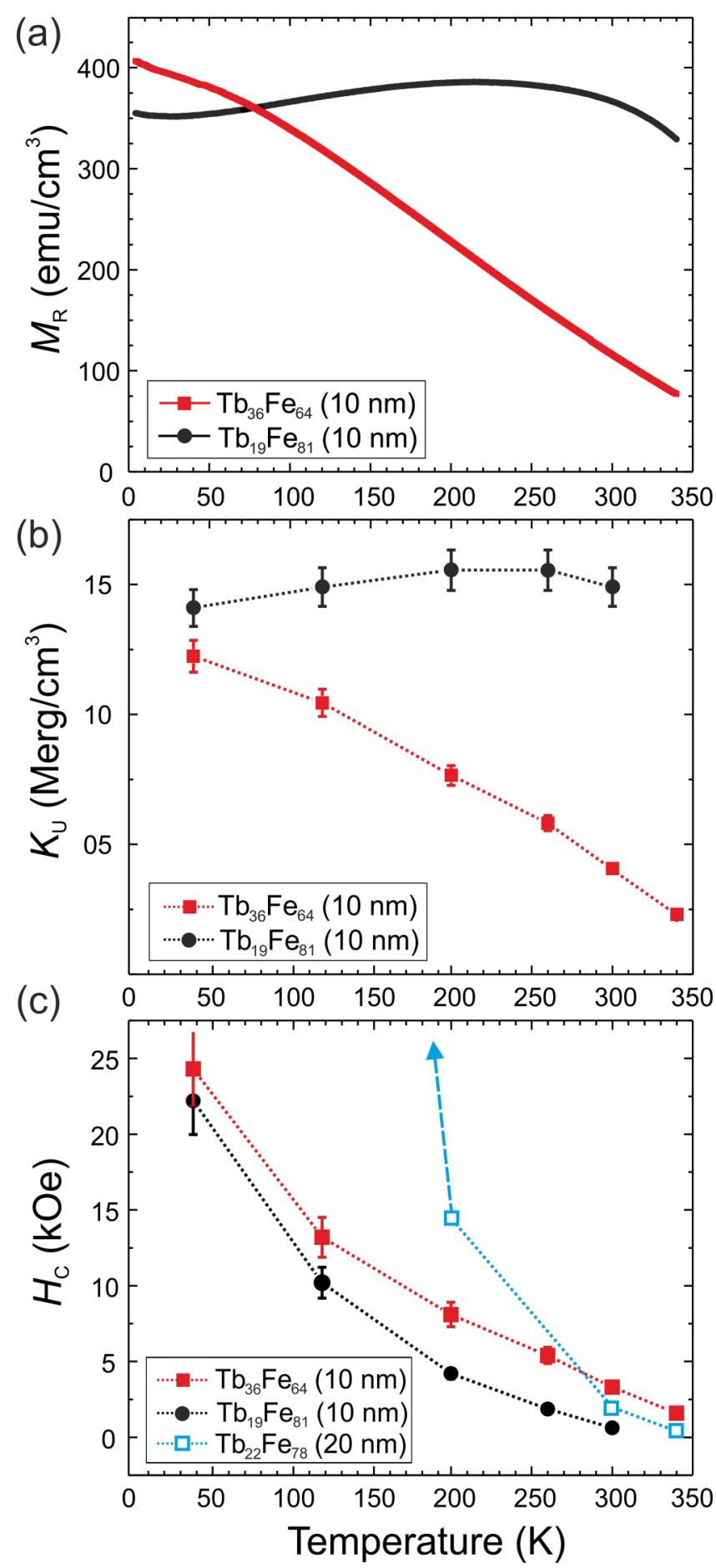

FIG. 1. (a) Remanent magnetization $M_{\mathrm{R}}$, (b) uniaxial magnetic anisotropy $K_{\mathrm{U}}$, and (c) coercivity $H_{\mathrm{C}}$ as a function of temperature measured for two 10-nm-thick Tb-Fe single layers. In (c), the temperature dependence of a 20 -nm-thick $\mathrm{Tb}_{22} \mathrm{Fe}_{78}$ single layer is included, where below $200 \mathrm{~K}$, the coercivity is larger than the accessible field range of $\pm 70 \mathrm{kOe}$.

both layers. In this regard, it is important to mention that the compensation temperature, remanent magnetization, and magnetic anisotropy of the magnetic layers depend not only on the composition but also strongly on the thickness of the magnetic film up to a critical thickness of about $20-30 \mathrm{~nm}$. 


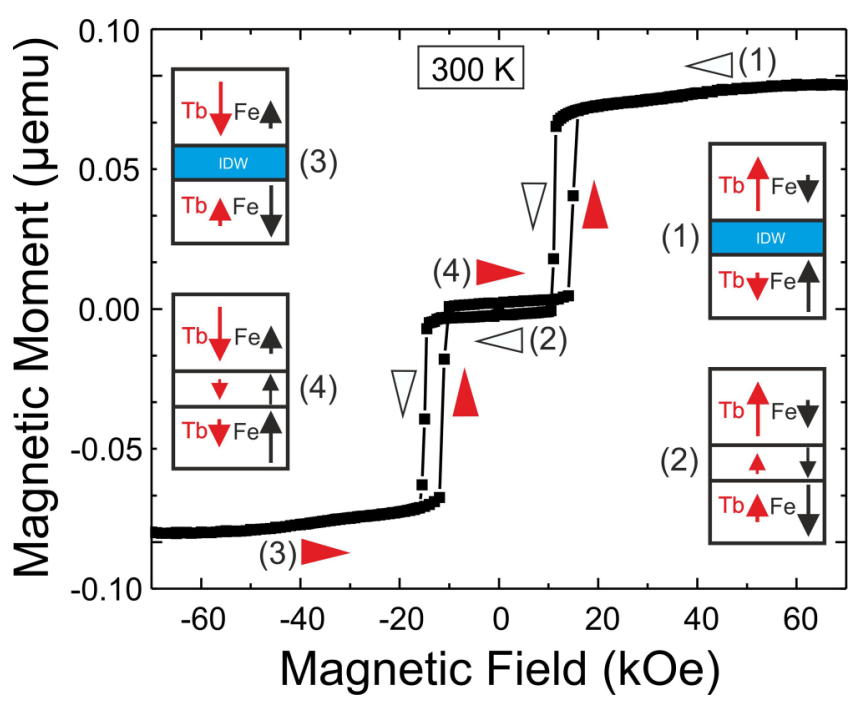

FIG. 2. (a) Out-of-plane $M$ - $H$ hysteresis loop of an antiferromagnetically coupled $\mathrm{Tb}_{19} \mathrm{Fe}_{81}(10 \mathrm{~nm}) / \mathrm{Tb}_{36} \mathrm{Fe}_{64}(10 \mathrm{~nm})$ heterostructure measured at room temperature. Included are the magnetic configurations during the reversal process.

Growth-induced variations in the short range order, resulting in a redistribution of the orientation of the $\mathrm{Tb}$ magnetic moments, are responsible for the thickness dependence, as discussed in detail in Ref. [32].

The out-of-plane $M-H$ hysteresis loop of the coupled $\mathrm{Tb}_{19} \mathrm{Fe}_{81}(10 \mathrm{~nm}) / \mathrm{Tb}_{36} \mathrm{Fe}_{64}(10 \mathrm{~nm})$ heterostructure at room temperature is presented in Fig. 2. The loop reveals the characteristic behavior of an antiferromagnetically coupled bilayer, as indicated by the reversal of the softer $\mathrm{Tb}_{19} \mathrm{Fe}_{81}$ layer before remanence after saturation in a positive field due to antiferromagnetic coupling of $\mathrm{Tb}$ and $\mathrm{Fe}$ moments of the two layers. As the $\mathrm{Tb}_{19} \mathrm{Fe}_{81}$ layer exhibits a slightly higher net magnetic moment compared to the $\mathrm{Tb}_{36} \mathrm{Fe}_{64}$ layer, a negative net moment at remanence is obtained [Fig 2, image 2]. This behavior is not expected, if one simply assumes a superposition of the magnetic moments of the individual layers shown in Fig. 1(a), where the moment of the $\mathrm{Tb}_{19} \mathrm{Fe}_{81}(10 \mathrm{~nm})$ layer is about three times larger than the moment of the $\mathrm{Tb}_{36} \mathrm{Fe}_{64}$ $(10 \mathrm{~nm})$ layer. This significant change in magnetization ratio, when comparing the bilayer system with the two single layer systems at room temperature, gives a first hint on the presence of a much more complex layer structure for the bilayer system, as will be discussed in more detail later.

In the following, we present the magnetic hysteresis loops of the $\mathrm{Tb}_{19} \mathrm{Fe}_{81}(10 \mathrm{~nm}) / \mathrm{Tb}_{36} \mathrm{Fe}_{64}(10 \mathrm{~nm})$ heterostructure taken at different temperatures and discuss the influence of cooling fields and different initial magnetization states on the reversal behavior.

In a first study, the sample was field cooled (FC) at $+70 \mathrm{kOe}$ down to $80 \mathrm{~K}$, and subsequently hysteresis loops were measured at various temperatures up to $300 \mathrm{~K}$. As shown in Fig. 3(a), a rather surprising result is obtained. The loop at $80 \mathrm{~K}$ reveals two exchange biased branches of different shape shifted in the positive $(\sim 10 \mathrm{kOe})$ and negative directions ( $\sim-50 \mathrm{kOe}$ ), which belong to the $\mathrm{Tb}$ - and Fe-dominated layer, respectively. The latter is verified by element-specific XMCD studies, as will be presented later. With increasing temperature, the EB shift gets slightly reduced and starts to vanish above $140 \mathrm{~K}$, as indicated by the appearance of a symmetric reversal part of the hysteresis between $\pm 15 \mathrm{kOe}$, which becomes more pronounced with increasing temperature. At $200 \mathrm{~K}$ and above, a fully symmetric hysteresis loop, characteristic of an antiferromagnetically coupled bilayer, as discussed before, is observed [11,14]. By cooling the system down to $80 \mathrm{~K}$ in a negative field of $-70 \mathrm{kOe}$ and measuring subsequently hysteresis loops from $80 \mathrm{~K}$ toward higher temperatures, the same evolution of the EB phenomenon is observed, except that the shifts are inverted [Fig. 3(b)]. Also, these loops do not show any notable change during consecutive loop cycling known as training effect [34]. In a further experiment, we prepared a demagnetized state, exhibiting large lateral domains in the size range of several micrometers, and $\mathrm{ZFC}$ down to $80 \mathrm{~K}$, which preserves the initial multidomain structure. Afterwards, subsequent hysteresis loops with increasing temperatures were measured. Interestingly, for this case, we just observe mainly the superposition of the behavior obtained after FC in positive and negative fields, as can be clearly seen, i.e. at $140 \mathrm{~K}$ [see Fig. 3(c)]. Therefore, the individually shifted branches, belonging to the $\mathrm{Tb}$ - and Fe-dominated layers, split equally into loops shifted in the positive and negative directions.

Furthermore, the evolution of the EB effect is rather independent from the thermomagnetic history below $140 \mathrm{~K}$. For instance, there is no change in loop shape whether loops were measure subsequently with increasing or decreasing temperature. Moreover, there is also no difference whether the loops are measured after FC at $70 \mathrm{kOe}$ or after ZFC when the sample was saturated at room temperature. However, the magnetic configuration during cooling is quite different in these two cases: While an IDW is present during FC at saturation, no IDW will be formed during ZFC at remanence [see Fig. 2]. Thus, we conclude that the EB effect itself is not related to an IDW formed at saturation during field cooling.

In order to get some more insight on the reversal mechanism of the individual layers in the $\mathrm{Tb}_{19} \mathrm{Fe}_{81}(10 \mathrm{~nm}) /$ $\mathrm{Tb}_{36} \mathrm{Fe}_{64}(10 \mathrm{~nm})$ heterostructure, element-specific XMCD hysteresis loops were measured at various temperatures. For these measurements, a comparable layer stack was deposited on $\mathrm{Si}_{3} \mathrm{~N}_{4}$ membranes using another sputter deposition chamber. Please note that the magnetic properties of this sample are slightly different compared to the film prepared on a $\mathrm{SiO}_{2} / \mathrm{Si}(001)$ substrate, due to different growth conditions. However, this sample reveals as well a double shifted loop confirming the reproducibility of this phenomenon.

In Fig. 4, element-specific XMCD hysteresis loops are shown for two different temperatures. By inspecting the polarity of the XMCD signal at the Fe and Tb absorption edges, opposite signs are observed, which shows that the magnetic moments of $\mathrm{Fe}$ and $\mathrm{Tb}$ are oriented antiparallel with respect to each other, demonstrating the FI nature of the layers. At $190 \mathrm{~K}$, the characteristic behavior of an antiferromagnetically coupled bilayer is observed. Coming from positive saturation, before remanence, the $\mathrm{Tb}_{19} \mathrm{Fe}_{81}$ layer, revealing a smaller $\mathrm{Tb}$ content (smaller XMCD Tb signal), reverses first, while the Tb-dominated layer reverses after applying a reverse field of about $-14 \mathrm{kOe}$. By $\mathrm{FC}$ in $80 \mathrm{kOe}$ and measuring the loop at $40 \mathrm{~K}$, we obtain the double shifted hysteresis loop, 


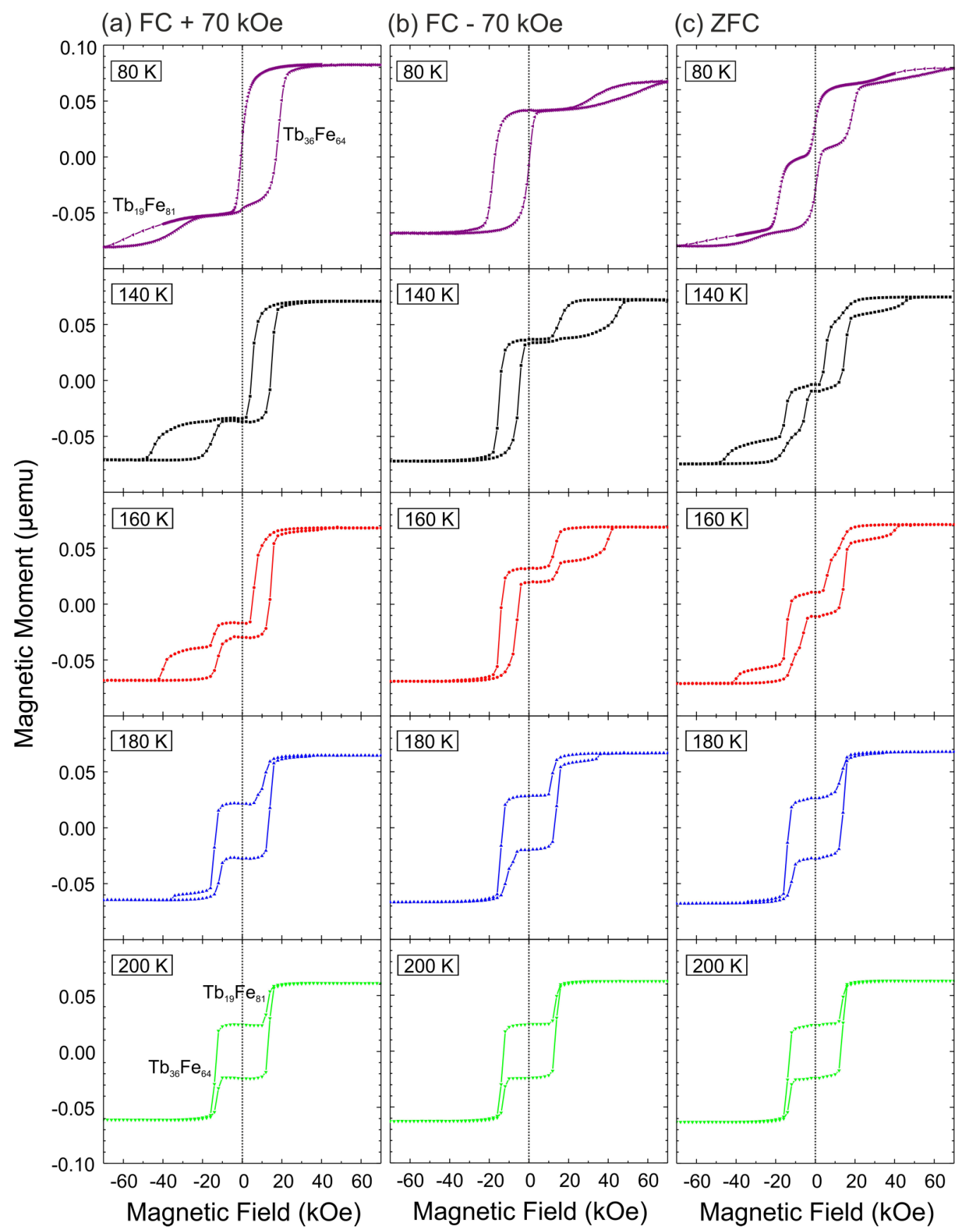

FIG. 3. Subsequent out-of-plane $M$ - $H$ hysteresis loops were measured at increasing temperatures after (a) FC (+70 kOe), (b) FC ( $-70 \mathrm{kOe})$, and (c) ZFC down to $80 \mathrm{~K}$. The measurements were performed on a $\mathrm{Tb}_{19} \mathrm{Fe}_{81}(10 \mathrm{~nm}) / \mathrm{Tb}_{36} \mathrm{Fe}_{64}(10 \mathrm{~nm})$ heterostructure.

confirming that the large positive EB effect corresponds to the Tb-dominated layer, while the negative EB effect is associated with the Fe-dominated layer.

Based on our experimental findings, the origin of this EB phenomenon can only be explained by the presence of an interfacial alloy layer, which has a larger coercivity than the maximum accessible field range below $160 \mathrm{~K}$, and thus can act as a pinning layer. This interfacial region needs to be $\mathrm{Fe}$ dominated. Only in this case, a positive (negative) EB effect can be obtained for the $\mathrm{Tb}(\mathrm{Fe})$ dominated layer, as observed experimentally by XMCD. If this interfacial region would be $\mathrm{Tb}$ dominated, then the corresponding loops would be shifted in the opposite directions. In addition, the presence of a compensation point is not required for the occurrence of the $\mathrm{EB}$ effect. It is assumed that this interfacial region is formed during the growth process due to interface mixing and re-sputtering effects, where for certain Tb-Fe compositions, hard magnetic properties can be achieved [32]. An example of a 20-nm-thick $\mathrm{Tb}_{22} \mathrm{Fe}_{78}$ single layer is included in Fig. 1(c), presenting the temperature dependence of the coercive field, which increases drastically towards lower temperatures and becomes larger than $70 \mathrm{kOe}$ below $200 \mathrm{~K}$. Please note that the formed interfacial region exhibits a variation of compositions, which vary locally, therefore the coexistence of pinned and unpinned 


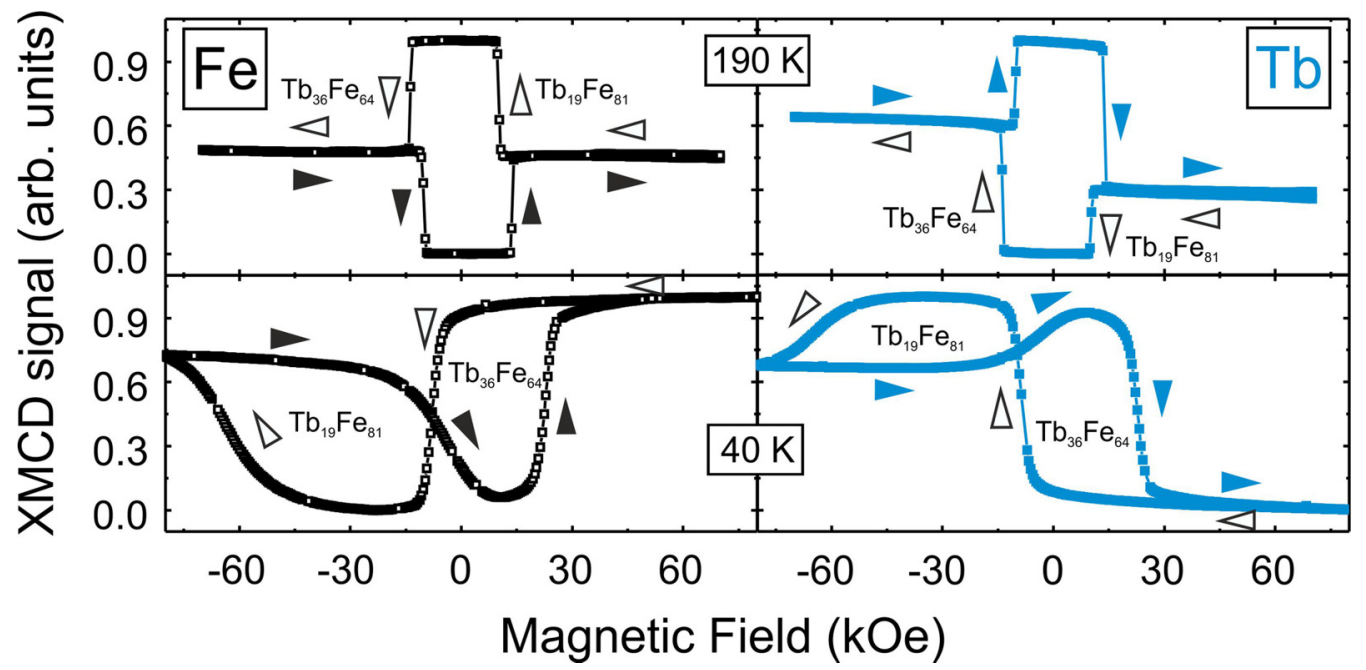

FIG. 4. Element-specific hysteresis loops of a $\mathrm{Tb}_{19} \mathrm{Fe}_{81}(10 \mathrm{~nm}) / \mathrm{Tb}_{36} \mathrm{Fe}_{64}(10 \mathrm{~nm})$ heterostructure obtained from XMCD absorption measurements at the $L_{3}$ edge of $\mathrm{Fe}(708 \mathrm{eV})$ and $M_{5}$ edge of $\mathrm{Tb}(1241 \mathrm{eV})$ at $190 \mathrm{~K}$ and $40 \mathrm{~K}$.

areas are expected. The relative portion between pinned and unpinned areas strongly depends on the temperature. By lowering the temperature down to $200 \mathrm{~K}$, more and more film area will be pinned, exhibiting exchange biased loops, while the unpinned film area will still reveal the characteristic reversal of the antiferromagnetically coupled bilayer. The superposition of pinned and unpinned film contributions can be clearly seen in the hysteresis loop obtained at $160 \mathrm{~K}$ [Fig. 3(a)]. Furthermore, by introducing a thin $0.9 \mathrm{~nm} \mathrm{Ru}$ spacer layer in the bilayer system, no EB shift occurs (not shown). This result supports the assumption that an interfacial region acting as a hard magnet is required to obtain exchange biased loops.

An illustration of the temperature-dependent magnetic reversal behavior of the coupled heterostructure, including the expected interfacial layer, is presented in Fig. 5. Here, we assume a multidomain state with lateral up and down domains, consisting of antiparallel aligned net magnetizations of the layers with an interfacial layer, which is Fe dominated [Fig. 5(a)]. By $\mathrm{ZFC}$, this multidomain configuration is preserved, but below $200 \mathrm{~K}$, some part of the interfacial layer starts to become magnetically hard and acts as a pinning layer. In Fig. 5(b), we consider only the case where the complete interfacial region is magnetically hard, as experimentally observed for temperatures below $140 \mathrm{~K}$. Rotation of the individual layers against the pinning direction results in a strong EB effect. Depending on the frozen magnetization orientation of the pinning layer, for the net down domains, the bias direction is positive for the $\mathrm{Tb}$-dominated layer and negative for the Fe-dominated layer, but inverted for net up domains. In both cases, a horizontal IDW between the pinning layer and the respective FI layer is nucleated and annihilated during reversal, as depicted in the corresponding images $(1,2)$ of Fig. 5(c). Accordingly, the appearance of equally distributed loop branches shifted in positive and negative directions, as observed experimentally in Fig. 3(c), can be explained. It should be mentioned that it is quite surprising that the vertical domain walls of the interfacial layer cannot be moved, even in applied fields of up to $80 \mathrm{kOe}$.

It is expected that the exchange coupling effect depends strongly on the composition variation and thickness range of the interfacial layer formed during the sputtering process. Consequently, the individual layer thicknesses are likely to have an important impact on this effect.

To elucidate this dependency, the magnetic reversal behavior of a $\mathrm{Tb}_{19} \mathrm{Fe}_{81}(20 \mathrm{~nm}-x) / \mathrm{Tb}_{36} \mathrm{Fe}_{64}(x)$ sample series with $x=5,7.5,10,12.5$, and $15 \mathrm{~nm}$ (nominal thicknesses) was analyzed. In this sample series, only the relative ratio in layer thickness of the bilayer was varied while keeping the total film thickness unchanged. The samples were FC at $+70 \mathrm{kOe}$, and then the hysteresis loops were measured at $300,200,160$, and $40 \mathrm{~K}$. Exemplarily, loops obtained at $160 \mathrm{~K}$ are shown in Fig. 6. For the $\mathrm{Tb}_{19} \mathrm{Fe}_{81} / \mathrm{Tb}_{36} \mathrm{Fe}_{64}$ (a) $\mathrm{T}>200 \mathrm{~K}$

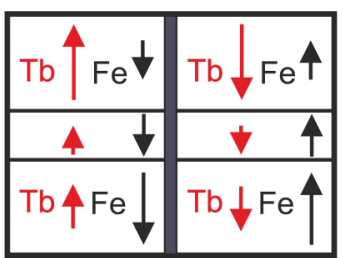

(b) $\mathrm{T}<140 \mathrm{~K}$

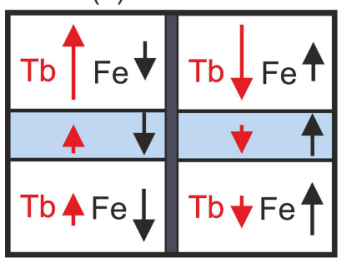

net domain up net domain down (c) $\mathrm{T}<140 \mathrm{~K}$, at saturation

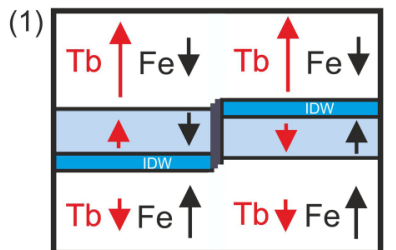

(2)

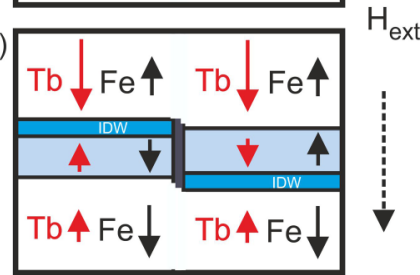

FIG. 5. (a) Illustration of a magnetic multidomain state at remanence above $200 \mathrm{~K}$. (b) Below $140 \mathrm{~K}$, the full interface layer becomes magnetically hard. (c) Rotation of the individual layers against the pinning direction results in a strong EB effect. For net down domains, its direction is positive for the Tb-dominated layer and negative for the Fe-dominated layer, but inverted for net up domains. In both cases, a horizontal IDW between the pinning layer and the respective FI layer will be nucleated and annihilated during reversal, as depicted in the corresponding images (1) and (2). 


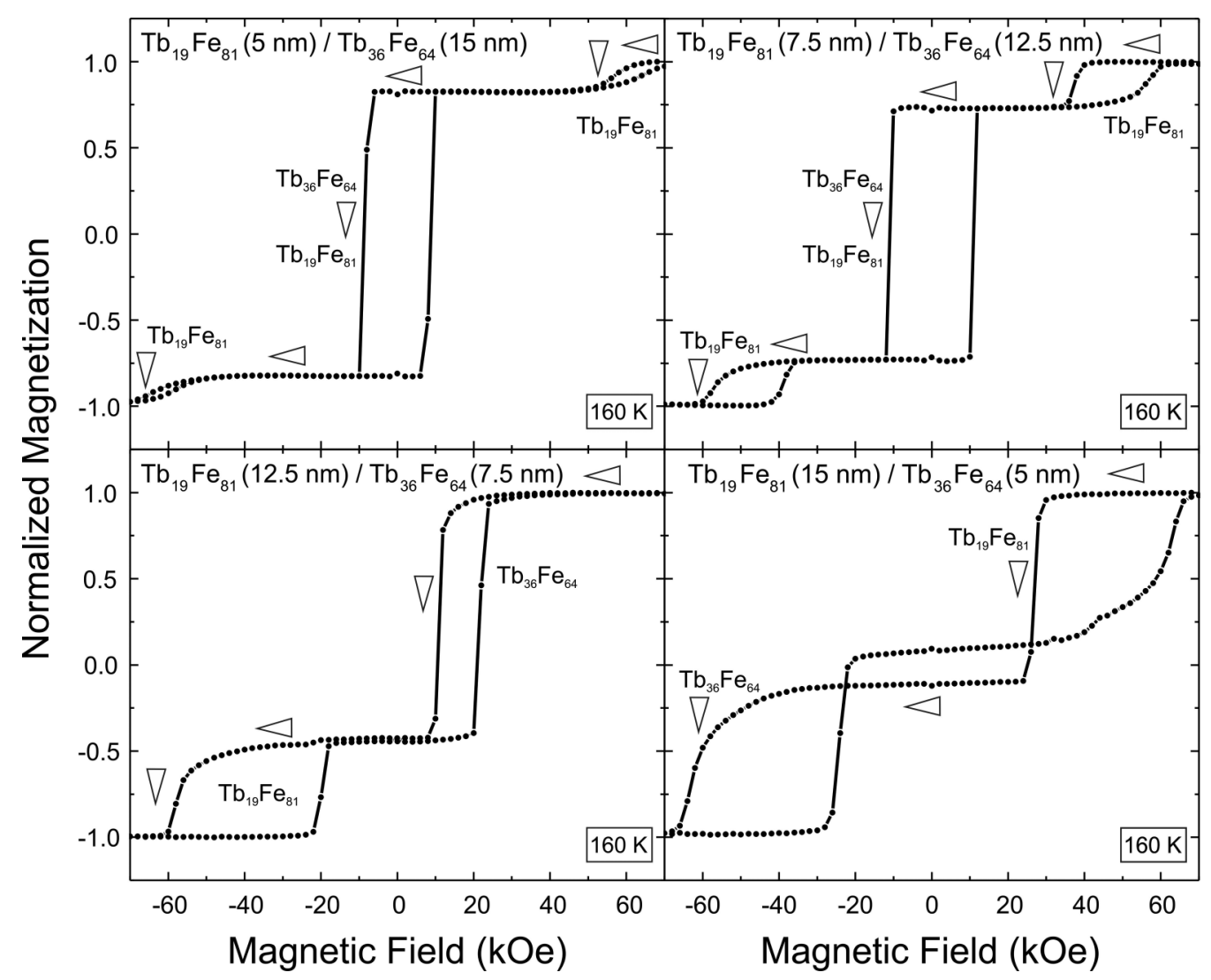

FIG. 6. Out-of-plane hysteresis loops of various $\mathrm{Tb}_{19} \mathrm{Fe}_{81} / \mathrm{Tb}_{36} \mathrm{Fe}_{64}$ bilayer samples measured at $160 \mathrm{~K}$ after $\mathrm{FC}$ at $+70 \mathrm{kOe}$.

heterostructures with $x=12.5$ and $15 \mathrm{~nm}$, a three-step reversal behavior is observed. In this case, starting from positive saturation, the thinner Fe-dominated ferrimagnet reverses before remanence (annihilation of the IDW). Then for a small negative reverse field, the coupled system reverses both their magnetization orientations, keeping the preferred antiparallel alignment until, at higher negative fields, the net magnetizations of the individual layers are aligned to the external field direction, thereby nucleating an IDW. For the sample with $x=5 \mathrm{~nm}$, a fully symmetric two-step reversal behavior characteristic for an antiferromagnetically coupled bilayer is observed. Only samples with $x=7.5$ and $10 \mathrm{~nm}$ reveal double shifted hysteresis loops, where the $\mathrm{Tb}$-dominated layer reverses first. Thus, it can be concluded that the difference in reversal behavior is based on a subtle balance between the different relevant energy contributions such as interfacial coupling strength, Zeeman energy, and magnetic anisotropy. Furthermore, the interfacial layer can only act as a pinning layer if its coercivity gets larger than the available field range (up to $\pm 80 \mathrm{kOe}$ ) at a certain temperature. Apparently, this perquisite depends strongly on the thickness of the FI layers, which is typically associated with a strong variation of the magnetic properties present in this thickness range [32]. Please note that, also, the order of the layer stack plays an important role. It is found that the Fe-dominated layer needs to be deposited on top of the Tb-dominated layer; otherwise, no double shifted loops will occur. This behavior indicates the strong dependence of the interfacial layer properties on interface mixing and resputtering effects during film growth.
In order to examine in more detail variations in composition with film thickness in particular at the interfacial region, SNMS depth profiles were analyzed for some selected samples of this series. As an example, the depth profile of the $\mathrm{Tb}_{19} \mathrm{Fe}_{81}(7.5 \mathrm{~nm}) / \mathrm{Tb}_{36} \mathrm{Fe}_{64}(12.5 \mathrm{~nm})$ sample, which does not exhibit any EB effect, is presented in Fig. 7. Please note that, for this sample, the $\mathrm{Tb}_{19} \mathrm{Fe}_{81}$ layer has a thickness of $6.5 \mathrm{~nm}$, deviating from the nominal thickness of $7.5 \mathrm{~nm}$, as

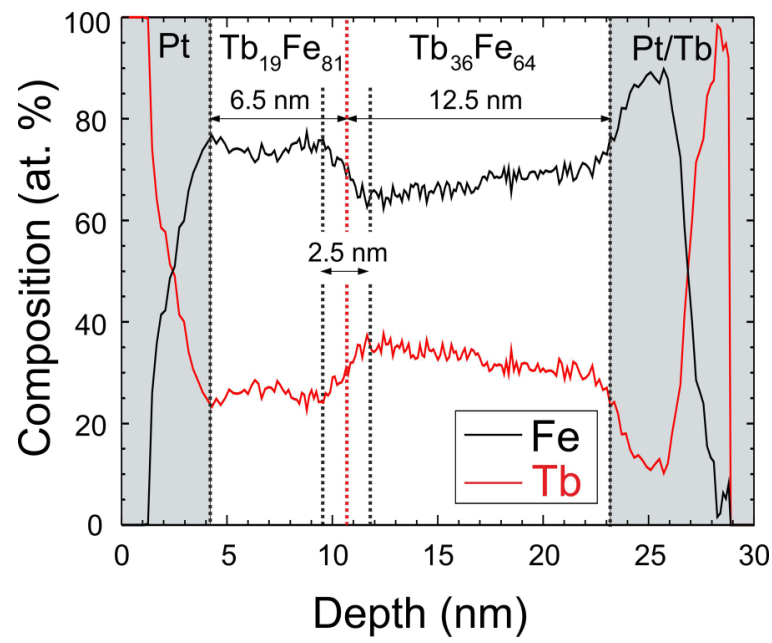

FIG. 7. SNMS depth profile of a $\mathrm{Pt}(5 \mathrm{~nm}) / \mathrm{Tb}_{19} \mathrm{Fe}_{81}(6.5 \mathrm{~nm}) /$ $\mathrm{Tb}_{36} \mathrm{Fe}_{64}(12.5 \mathrm{~nm}) / \mathrm{Pt}(5 \mathrm{~nm}) / \mathrm{Tb}(2 \mathrm{~nm}) / \quad \mathrm{SiO}_{2}(100 \mathrm{~nm}) / \mathrm{Si}(001)$ sample. 
determined by SNMS and verified by RBS. Most interestingly, a 2-3-nm-thick interfacial region, where the composition changes continuously between the two FI layers is observed. However, in the depth profile close to the Pt seed and cover layer, a strong matrix effect, influencing drastically the $\mathrm{Fe}$ and $\mathrm{Tb}$ yield, is observed, which does not allow drawing any clear conclusion about the $\mathrm{Tb}$ or Fe contents. Nonetheless, throughout the sample series, very similar depth profiles were obtained, revealing no noticeable difference in particular at the interface region.

\section{CONCLUSIONS}

In conclusion, a rather peculiar exchange coupling phenomenon was observed in amorphous $\mathrm{FI} \mathrm{Tb}{ }_{19} \mathrm{Fe}_{81} / \mathrm{Tb}_{36} \mathrm{Fe}_{64}$ heterostructures, consisting of $\mathrm{Tb}$ - and Fe-dominated FI layers. At remanence, the net magnetic moments of the individual layers are oppositely aligned due to antiferromagnetic coupling of $\mathrm{Fe}$ and $\mathrm{Tb}$ moments. In addition, there is evidence that a Fe-dominated interface alloy is present due to interface mixing and resputtering effects during film growth, exhibiting large coercivity and exceeding the accessible field range up to $80 \mathrm{kOe}$ below $160 \mathrm{~K}$. As a result, this interface layer does act as a pinning layer for the two ferrimagnets. Rotation of the individual layers against the pinning direction results in a strong loop shift, which is positive for the Tb-dominated layer and negative for the Fe-dominated layer, as verified by element-specific XMCD studies. This assumption is further supported by introducing a $\mathrm{Ru}$ spacer layer, where the EB effect is eliminated.

In a further study, the thickness ratio of the two $\mathrm{Tb}-\mathrm{Fe}$ layers was varied, keeping the total thickness unchanged. Only samples with sufficiently thick individual layers reveal double shifted loops. This dependence can be explained by the change in magnetic properties of the individual layers, including the interfacial layer. Hence, there seems to be a subtle balance between all these properties across the interface region and their contribution to this rather unexpected exchange coupling phenomenon. Based on our findings and understanding, in future studies, we plan to explore corresponding trilayer systems, where the thickness and composition of the naturally formed interlayer phase is controlled and tuned directly by the deposition parameters, allowing for specific applications where large EB effects are needed.

\section{ACKNOWLEDGMENTS}

This work was financially supported by the Deutsche Forschungsgemeinschaft (Grant No. AL618/24-1) and by a Bavaria California Technology Center $(\mathrm{BaCaTeC})$ grant to B.H. The authors are thankful for the allocation of synchrotron radiation beam time and acknowledge the financial support from HZB (Project No. 16103747-ST). In particular, we thank Florin Radu and Chen Luo for beamline assistance.
[1] W. H. Meiklejohn and C. P. Bean, Phys. Rev. 102, 1413 (1956).

[2] I. L. Prejbeanu, S. Bandiera, J. Alvarez-Hérault, R. Sousa, B. Dieny, and J. P. Nozières, J. Phys. D. Appl. Phys. 46, 074002 (2013).

[3] S. Roy, M. R. Fitzsimmons, S. Park, M. Dorn, O. Petracic, I. V. Roshchin, Z. P. Li, X. Batlle, R. Morales, A. Misra, X. Zhang, K. Chesnel, J. B. Kortright, S. K. Sinha, and I. K. Schuller, Phys. Rev. Lett. 95, 047201 (2005).

[4] F. Radu and H. Zabel, Springer Tracts Mod. Phys. 227, 97 (2008).

[5] K. O'Grady, L. Fernandez-Outon, and G. Vallejo-Fernandez, J. Magn. Magn. Mater. 322, 883 (2010).

[6] I. Schmid, M. A. Marioni, P. Kappenberger, S. Romer, M. Parlinska-Wojtan, H. J. Hug, O. Hellwig, M. J. Carey, and E. E. Fullerton, Phys. Rev. Lett. 105, 197201 (2010).

[7] H. Ohldag, T. J. Regan, J. Stöhr, A. Scholl, F. Nolting, J. Lüning, C. Stamm, S. Anders, and R. L. White, Phys. Rev. Lett. 87, 247201 (2001).

[8] H. Ohldag, A. Scholl, F. Nolting, E. Arenholz, S. Maat, A. T. Young, M. Carey, and J. Stöhr, Phys. Rev. Lett. 91, 017203 (2003).

[9] J. Wu, J. S. Park, W. Kim, E. Arenholz, M. Liberati, A. Scholl, Y. Z. Wu, C. Hwang, and Z. Q. Qiu, Phys. Rev. Lett. 104, 217204 (2010).

[10] F. Canet, S. Mangin, C. Bellouard, and M. Piecuch, Europhys. Lett. 52, 594 (2000).

[11] S. Mangin, T. Hauet, P. Fischer, D. H. Kim, J. B. Kortright, K. Chesnel, E. Arenholz, and E. E. Fullerton, Phys. Rev. B 78, 024424 (2008).
[12] M. Watson, T. Hauet, J. A. Borchers, S. Mangin, and E. E. Fullerton, Appl. Phys. Lett. 92, 202507 (2008).

[13] S. Romer, M. A. Marioni, K.Thorwarth, N. R. Joshi, C. E. Corticelli, H. J. Hug, S. Oezer, M. Parlinska-Wojtan, and H. Rohrmann, Appl. Phys. Lett. 101, 222404 (2012).

[14] C. Schubert, B. Hebler, H. Schletter, A. Liebig, M. Daniel, R. Abrudan, F. Radu, and M. Albrecht, Phys. Rev. B 87, 054415 (2013).

[15] M. Patra, M. Thakur, S. Majumdar, and S. Giri, J. Phys.: Condens. Matter 21, 236004 (2009).

[16] M. Ungureanu, K. Dumesnil, C. Dufour, N. Gonzalez, F. Wilhelm, A. Smekhova, and A. Rogalev, Phys. Rev. B 82, 174421 (2010).

[17] S. Mangin, F. Montaigne, and A. Schuhl, Phys. Rev. B 68 , 140404 (2003).

[18] T. Hauet, S. Mangin, F. Montaigne, J. A. Borchers, and Y. Henry, Appl. Phys. Lett. 91, 022505 (2007).

[19] F. Radu, R. Abrudan, I. Radu, D. Schmitz, and H. Zabel, Nat. Commun. 3, 715 (2012).

[20] C.-C. Lin, C.-H. Lai, R.-F. Jiang, and H.-P. D. Shieh, J. Appl. Phys. 93, 6832 (2003).

[21] S. Mangin, T. Hauet, Y. Henry, F. Montaigne, and E. E. Fullerton, Phys. Rev. B 74, 024414 (2006).

[22] T. Hauet, F. Montaigne, M. Hehn, Y. Henry, and S. Mangin, Phys. Rev. B 79, 224435 (2009).

[23] T. Tokunaga, M. Taguchi, T. Fukami, and K. Tsutsumi, J. Appl. Phys. 67, 4417 (1990)

[24] M. H. Tang, Z. Zhang, S. Y. Tian, J. Wang, B. Ma, and Q. Y. Jin, Sci. Rep. 5, 10863 (2015). 
[25] M. H. Tang, Z. Zhang, Y. Zu, B. Ma, and Q. Y. Jin, Nano-Micro Lett. 6, 359 (2014).

[26] B. Hebler, S. Böttger, D. Nissen, R. Abrudan, F. Radu, and M. Albrecht, Phys. Rev. B 93, 184423 (2016).

[27] E. E. Fullerton, J. S. Jiang, M. Grimsditch, C. H. Sowers, and S. D. Bader, Phys. Rev. B 58, 12193 (1998).

[28] K. G. West, D. H. N. Nam, J. W. Lu, N. D. Bassim, Y. N. Picard, R. M. Stroud, and S. A. Wolf, J. Appl. Phys. 107, 113915 (2010).

[29] A. K. Nayak, M. Nicklas, S. Chadov, P. Khuntia, C. Shekhar, A. Kalache, M. Baenitz, Y. Skourski, V. K. Guduru, A. Puri, U.Zeitler, J. M. D. Coey, and C. Felser, Nat. Mater. 14, 679 (2015).
[30] H. Oechsner, R. Getto, and M. Kopnarski, J. Appl. Phys. 105, 063523 (2009).

[31] T. Funk, A. Deb, S. J. George, H. Wang, and S. P. Cramer, Coord. Chem. Rev. 249, 3 (2005).

[32] B. Hebler, A. Hassdenteufel, P. Reinhardt, H. Karl, and M. Albrecht, Front. Mater. 3, 8 (2016).

[33] C. Schubert, A. Hassdenteufel, P. Matthes, J. Schmidt, M. Helm, R. Bratschitsch, and M. Albrecht, Appl. Phys. Lett. 104, 082406 (2014).

[34] T. Hauet, J. A. Borchers, P. Mangin, Y. Henry, and S. Mangin, Phys. Rev. Lett. 96, 067207 (2006). 